\title{
Publier en français : why not ?
}

\section{Publish in French: Pourquoi pas?}

\author{
Y. Touitou \\ (C) Lavoisier SAS 2016
}

Impact factor (IF), points SIGAPS (Système d'interrogation, de gestion et d'analyse des publications scientifiques), points SIAPS (Score individuel d'aptitudes pédagogiques en santé), des acronymes que les hospitalo-universitaires connaissent bien parce que ces termes sont des sésames qui jalonnent leurs parcours lors des nominations ou des promotions de leurs activités. De quoi s'agit-il ? Le facteur d'impact, plus connu sous sa désignation anglaise d'impact factor, est un outil bibliométrique, une note attribuée à un journal scientifique prenant en compte, chaque année, le nombre de citations du journal par la littérature scientifique mondiale. Plus la note est élevée plus la revue est recherchée car considérée comme prestigieuse. Les revues anglosaxonnes tiennent le haut du pavé, pour en citer quelques unes : NEJM, Nature, Science, Lancet, et il y en a bien d'autres naturellement. Cet IF sert lui-même au calcul des points SIGAPS, un score d'évaluation de l'activité scientifique qui servira à la nomination/promotion des candidats. Les jurys des CNU sont attentifs à ces scores dans leurs évaluations des dossiers scientifiques et par voie de conséquence les hospitalo-universitaires favorisent très largement les revues anglosaxonnes pour la publication de leurs résultats scientifiques. Les revues de langue française sont donc délaissées et un certain nombre d'entre elles ont périclité.

Il est vain de penser que cette situation peut s'inverser alors qu'il est par ailleurs primordial de redonner une chance aux revues médicales de langue française et de les voir s'épanouir à nouveau. La seule possibilité est de trouver un moyen d'incitation, presque une obligation, à publier en français. La conférence des doyens des Facultés de médecine et la conférence des présidents du Conseil national des universités (CNU), conscientes de l'importance de soutenir la langue française en médecine au service des médecins français et de l'ensemble des médecins francophones, ont imaginé une sorte de jumeau du SIGAPS appelé SIAPS, l'APS du premier correspondant à l'Analyse des publications scientifiques, l'APS du second correspondant à l'Aptitude pédagogique en santé. Ce score a vocation pour quantifier l'implication des candidats dans le champ de la pédagogie. Outre l'évaluation de l'activité scientifique, l'activité pédagogique des candidats, en particulier les cours et les enseignements dirigés auxquels ils participent seront évalués comme le seront les activités de publications en langue française qui pourraient comporter par exemple des revues générales ou des commentaires. Les CNU seront alors tout aussi attentifs à l'évaluation de l'activité d'enseignement rédactionnel qu'ils le sont dans l'évaluation scientifique des candidats. L'Académie nationale de médecine vient, dans un communiqué que vous trouverez à gauche de cet éditorial, d'apporter son soutien à cette initiative tout en soulignant la nécessité d'une simplification du projet actuel de grille et de sa modulation par les différentes sous-sections du CNU en fonction de leurs objectifs pédagogiques propres.

Y. Touitou $(\square)$

Unité de chronobiologie, Fondation A. de Rothschild,

F-75019 Paris, France

e-mail : yvan.touitou@chronobiology.fr 